\title{
An efficient synthesis of a hexasaccharide-the repeating unit of the exopolysaccharide from Cryptococcus neoformans serovar A
}

\author{
Jianjun Zhang and Fanzuo Kong* \\ Research Center for Eco-Environmental Sciences, Academia Sinica, PO Box 2871, Beijing 100085, P.R. China
}

Received 11 November 2002; revised 3 January 2003; accepted 10 January 2003

\begin{abstract}
A general method for the synthesis of 2-xylose or glucuronic acid branched (1 $\rightarrow 3)$-linked mannose oligosaccharides has been developed. As a typical example, the synthesis of the methyl glycoside of $\beta$-D-Glc $p$ A- $(1 \rightarrow 2)-\alpha-\mathrm{D}-\mathrm{Man} p-(1 \rightarrow 3)-[\beta-\mathrm{D}-\mathrm{Xyl} p-(1 \rightarrow$ $2)-] \alpha-\mathrm{D}-\operatorname{Man} p-(1 \rightarrow 3)-[\beta-\mathrm{D}-\mathrm{Xyl} p-(1 \rightarrow 2)-] \alpha-\mathrm{D}-\operatorname{Man} p$, the repeating unit of the exopolysaccharide from Cryptococcus neoformans serovar A, was achieved in a regio- and stereoselective manner. (C) 2003 Elsevier Science Ltd. All rights reserved.
\end{abstract}

Cryptococcus neoformans has been a primary cause of opportunistic infections in patients diagnosed with AIDS. ${ }^{1}$ The yeast $C$. neoformans is a pulmonary pathogen and can disseminate to the central nervous system causing meningoencephalitis. ${ }^{2} C$. neoformans produces large amounts of a polysaccharide composed of mannose, xylose, and glucuronic acid. This polysaccharide, termed glucuronoxylomannan $(\mathrm{GXM}){ }^{3}$ is the major constituent of the cryptococcal capsule. ${ }^{4}$

GXM occurs as four major serotypes, ${ }^{5}$ designated A-D (Scheme 1). All the four serotypes have a linear $\alpha-1,3-$ linked mannosyl backbone with $\beta$-glucopyranosyluronic acid and $\beta$-xylopyranosyl substituents. ${ }^{6-9}$ In addition, the backbone is substituted with variable amounts of 6-O-acetyl groups, with serotype $\mathrm{D}$ being the most heavily $O$-acetylated and serotype $\mathrm{C}$ the least $O$-acetylated. ${ }^{10}$ However, the $O$-acetyl groups are not essential for binding, though they have a significant contribution. ${ }^{3}$

Synthesis of the repeating units of the polysaccharide from $C$. neoformans serotypes $\mathrm{A}-\mathrm{D}$ is of interest since it can afford synthetic samples in sufficient amounts for studies on the relationship of oligosaccharide structurebioactivity. However, this synthesis is not easy because of the relatively poor reactivity of the 2-axial hydroxy group of mannose, and the steric hindrance caused by the 2,3-substitution of the mannose residues. The synthesis of trisaccharide and tetrasaccharide fragments corresponding to structures in capsular polysaccharides of $C$. neoformans has been reported ${ }^{11,12}$ and the synthesis of a pentasaccharide - the repeating unit of the polysaccharide in $C$. neoformans serovar $\mathrm{D}$ has appeared. ${ }^{13}$ In these syntheses, multiple steps and orthogonal masking groups were involved making the

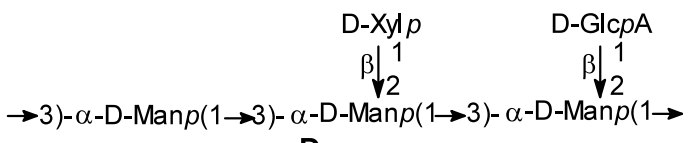

D

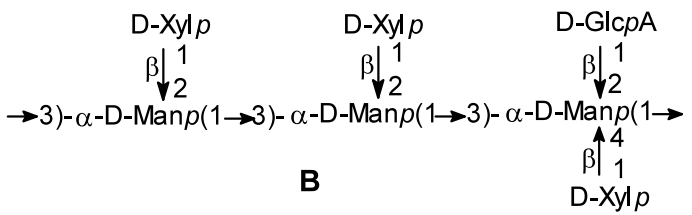

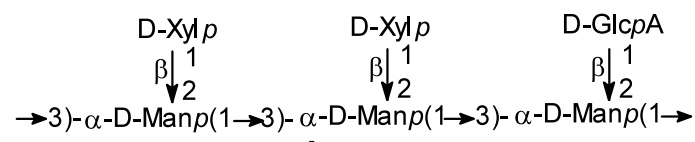

A

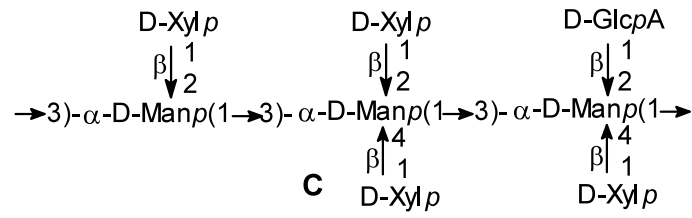

Scheme 1. Model structures of GXM of C. neoformans serotypes A-D.

\footnotetext{
* Corresponding author. 

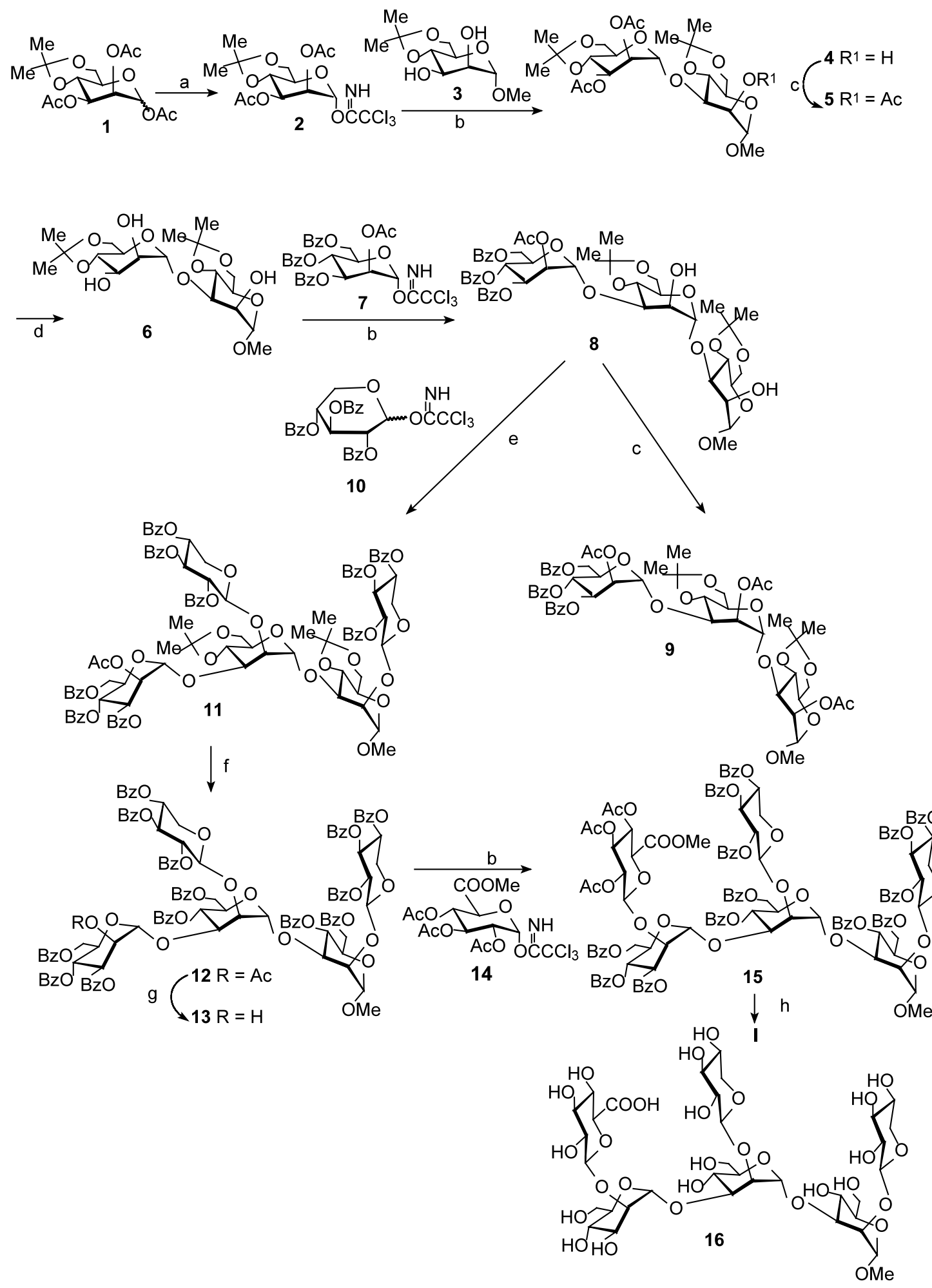

Scheme 2. Reagents and conditions: (a) (i) THF-CH $\mathrm{CH}_{3} \mathrm{OH}, 1.5 \mathrm{~N} \mathrm{NH}_{3}, \mathrm{rt}, 2-3 \mathrm{~h}$; (ii) $\mathrm{CH}_{2} \mathrm{Cl}_{2}, \mathrm{CCl}_{3} \mathrm{CN}$ (2.0 equiv.), $\mathrm{K}_{2} \mathrm{CO}_{3}(2.0$ equiv.), rt, $12 \mathrm{~h}, 71 \%$; (b) TMSOTf (0.01 equiv.), $4 \AA \mathrm{MS}, \mathrm{CH}_{2} \mathrm{Cl}_{2},-20^{\circ} \mathrm{C}, 2-4 \mathrm{~h}$ (74\% for 4, $67 \%$ for $\mathbf{8}, 88 \%$ for 15); (c) $\mathrm{Ac}_{2} \mathrm{O}$-pyridine, $100 \%$; (d) $\mathrm{CH}_{3} \mathrm{OH}$ saturated with ammonia, rt, $12 \mathrm{~h}, 100 \%$; (e) $\mathbf{1 0}$ (2 equiv.), TMSOTf (0.1 equiv.), $4 \AA \mathrm{MS}$, $\mathrm{CH}_{2} \mathrm{Cl}_{2},-0^{\circ} \mathrm{C}, 0.5 \mathrm{~h}$; then TMSOTf (1.0 equiv.), $0.5 \mathrm{~h}$, and further 10 (2 equiv.) was added, $42 \%$; (f) (i) $90 \% \mathrm{HOAc}, 60^{\circ} \mathrm{C}, 20 \mathrm{~h}$; (ii) $\mathrm{BzCl}$-pyridine, rt, $10 \mathrm{~h}, 91 \%$; (g) 2\% $\mathrm{CH}_{3} \mathrm{COCl}$ in $\mathrm{CH}_{2} \mathrm{Cl}_{2}-\mathrm{CH}_{3} \mathrm{OH}, \mathrm{rt}, 20 \mathrm{~h}, 67 \%$; (h) methanol saturated with ammonia, rt, $36 \mathrm{~h}$, then water (2 equiv.) was added, $5 \mathrm{~h}, 65 \%$. 
procedure rather complex. As a result, it would be difficult to synthesize by the reported methods, the higher oligosaccharides - the repeating units of $C$. neoformans serotypes A, B, and C. Previously, we have reported the regio- and stereoselective synthesis of oligosaccharides with un- or lightly protected mannose ${ }^{14}$ and rhamnose $e^{15}$ as the glycosyl acceptors and glycosyl trichloroacetimidates as the donors giving satisfactory results. We present herein, for the first time, the regio- and stereoselective synthesis of a frameshifted hexasaccharide repeating unit ${ }^{16}$ I (structure $\mathbf{1 6}$ in Scheme 2) of $O$-deacetylated GXM of $C$. neoformans serotype A with lightly protected mannose derivatives as the acceptors.

As shown in Scheme 2, 1,2,3-tri- $O$-acetyl-4,6- $O$-isopropylidene-D-mannopyranose $\mathbf{1}$, obtained from selective 4,6- $O$-isopropylidenation of mannose $^{17}$ with 2-methoxypropene followed by acetylation, was chosen as the starting material. Selective 1-O-deacetylation with ammonia in $\mathrm{THF}-\mathrm{MeOH}$ followed by trichloroacetimidation ${ }^{18}$ with trichloroacetonitrile in the presence of potassium carbonate gave the donor 2,3di- $O$-acetyl-4,6- $O$-isopropylidene- $\alpha$-D-mannopyranosyl trichloroacetimidate 2. Condensation of $\mathbf{2}$ with the acceptor methyl 4,6- $O$-isopropylidene- $\alpha$-D-mannopyranoside 3 selectively afforded the $(1 \rightarrow 3)$-linked disaccharide $4(74 \%)$. The regioselectivity of the coupling was confirmed by acetylation of $\mathbf{4}$ to give 5 , and the ${ }^{1} \mathrm{H}$ NMR spectrum of $\mathbf{5}$ showed a newly emerged downfield doublet of doublets at $\delta 5.34 \mathrm{ppm}$ with $J_{1,2}=1.5$ and $J_{2,3}=3.0 \mathrm{~Hz}$ for $\mathrm{H}-2$ compared to that of 4. Deacetylation of $\mathbf{4}$ or $\mathbf{5}$ in a solution of ammonia in methanol furnished the disaccharide triol acceptor 6 quantitatively. Again, 3-O-selective glycosylation of $\mathbf{6}$ with the donor 2- $O$-acetyl-3,4,6-tri- $O$-benzoyl- $\alpha$-Dmannopyranosyl trichloroacetimidate 7 yielded $(1 \rightarrow 3)$ linked trisaccharide $8(67 \%)$. Confirmation of the 3-regioselectivity was carried out by acetylation of $\mathbf{8}$ to give the trisaccharide 9 which showed two characteristic signals at $\delta 5.40 \mathrm{ppm}$ with $J_{1,2}=1.3, J_{2,3}=3.4 \mathrm{~Hz}$ for $\mathrm{H}-2$, and $5.22 \mathrm{ppm}$ with $J_{1,2}=1.3, J_{2,3}=2.9 \mathrm{~Hz}$ for $\mathrm{H}-2^{\prime}$, respectively. The trisaccharide $\mathbf{8}$ was an ideal acceptor since it contained two free hydroxyl groups at the positions where the xylosyl residue should be attached. Thus, TMSOTf promoted xylosylation of 8 with 2,3,4tri- $O$-benzoyl-D-xylopyranosyl trichloroacetimidate $\mathbf{1 0}$ was carried out. It was noted that at the initial stage of the coupling, formation of the tetrasaccharide intermediate was quite fast and little pentasaccharide was obtained. The quantity of pentasaccharide did not increase along with the reaction time. For completion of the reaction, an 'inverse Schmidt' strategy was used. Thus, further TMSOTf was added, and after stirring the reaction mixture for $20 \mathrm{~min}$, further 2 equiv. of donor $\mathbf{1 0}$ was added giving the pentasaccharide $\mathbf{1 1}$ in fair yield (42\%). De-isopropylidenation of 11 in $60 \%$ aqueous acetic acid at $60^{\circ} \mathrm{C}$ followed by benzoylation with benzoyl chloride in pyridine gave the protected pentasaccharide 12. Selective deacetylation of 12 with $2 \%$ acetyl chloride-methanol ${ }^{19}$ was accompanied by some decomposition, perhaps caused by breaking of xylosyl linkage, giving the pentasaccharide acceptor $\mathbf{1 3}$ in $67 \%$ yield. Coupling of 13 with methyl 2,3,4-tri- $O$ acetyl- $\alpha$-D-glucopyranosyluronate trichloroacetimidate 14 went smoothly affording the protected hexasaccharide in good yield (88\%). The ${ }^{1} \mathrm{H}$ NMR spectrum of $\mathbf{1 5}$ showed two methyl signals $(\delta 3.69 \mathrm{ppm}$ and $3.23 \mathrm{ppm}$, respectively), thirteen benzoyl $\mathrm{C}=\mathrm{O}$ signals $(\delta$ 165.9, $165.9,165.9,165.4,165.4,165.4,165.3,165.2,165.1$, $165.0,164.9,164.6,164.6 \mathrm{ppm})$, four $\mathrm{C}=\mathrm{O}$ signals $(\delta$ $167.0,168.5,168.5,168.3 \mathrm{ppm})$, six anomeric $\mathrm{C}$ signals $\left(100.9, J_{\mathrm{C} 1, \mathrm{H} 1}=175 \mathrm{~Hz}, \operatorname{Man} p ; 100.3, J_{\mathrm{C} 1, \mathrm{H} 1}=163 \mathrm{~Hz}\right.$, GluAp; 99.9, $J_{\mathrm{C} 1, \mathrm{H} 1}=164 \mathrm{~Hz}, \mathrm{Xyl} p ; 99.5, J_{\mathrm{C} 1, \mathrm{H} 1}=163$ $\mathrm{Hz}, \mathrm{Xyl} p ; 98.5, J_{\mathrm{C} 1, \mathrm{H} 1}=172 \mathrm{~Hz}, \operatorname{Man} p ; 95.2, J_{\mathrm{C} 1, \mathrm{H} 1}=$ $176 \mathrm{~Hz}$, Man $p$ ). ${ }^{20}$ Deprotection of 15 was carried out in a saturated solution of ammonia in methanol for $36 \mathrm{~h}$, then water ( 2 equiv.) was added to cleave the methyl ester. After standing at room temperature for $5 \mathrm{~h}$, the reaction mixture was concentrated and purified on a Bio-Gel P2 column (eluent: water), affording the target hexasaccharide $\mathbf{1 6}$ as a foamy solid.

In summary, an efficient synthesis of a hexasaccharide repeat unit of $O$-deacetylated GXM of $C$. neoformans serotype A with lightly protected mannose derivatives as the acceptors was achieved. The strategy presented here also provides a route to the synthesis of more complex repeating units of GXM of $C$. neoformans serotype $\mathrm{B}$ and $\mathrm{C}$.

\section{Acknowledgements}

This work was supported by The Chinese Academy of Sciences (KZCX3-J-08) and by The National Natural Science Foundation of China (Projects 30070185 and 39970864).

\section{References}

1. Dismukes, W. E. J. Infect. Dis. 1988, 157, 624.

2. Diamond, R. D. In Principles of Infectious Diseases; Mandell, G. L.; Douglas, R. G., Jr.; Bennett, J. E., Eds.; Churchhill Livingstone: New York, 1990; pp. 1980-1989.

3. Otteson, E. W.; Welch, W. H.; Kozel, T. R. J. Biol. Chem. 1994, 269, 1858

4. Cherniak, R.; Reiss, E.; Slodki, M. E.; Plattner, R. D.; Blumer, S. O. Mol. Immunol. 1980, 17, 1025.

5. Wilson, D. E.; Bennett, J. E.; Bailey, J. W. Proc. Soc. Exp. Biol. Med. 1968, 127, 820.

6. Bhattacharjee, A. K.; Kwon-Chung, K. J.; Glaudemans, C. P. J. Carbohydr. Res. 1979, 73, 183.

7. Bhattacharjee, A. K.; Kwon-Chung, K. J.; Glaudemans, C. P. J. Carbohydr. Res. 1981, 95, 237.

8. Bhattacharjee, A. K.; Kwon-Chung, K. J.; Glaudemans, C. P. J. Carbohydr. Res. 1980, 82, 103.

9. Bhattacharjee, A. K.; Kwon-Chung, K. J.; Glaudemans, C. P. J. Mol. Immunol. 1979, 16, 531.

10. Kozel, T. R.; Hermerath, C. A. Infect. Immun. 1984, 43, 879 .

11. Garegg, P. J.; Olsson, L.; Oscarson, S. J. Carbohydr. Chem. 1993, 12, 195. 
12. Garegg, P. J.; Olsson, L.; Oscarson, S. Bioorg. Med. Chem. 1996, 4, 1867.

13. ZegelaarJaarsveld, K.; Smits, S. A. W.; van der Marel, G. A.; van Boom, J. H. Bioorg. Med. Chem. 1996, 4, 1819.

14. (a) Zhu, Y.; Kong, F. Synlett 2001, 1217; (b) Zhu, Y.; Kong, F. Synlett 2000, 663; (c) Zhang, J.; Kong, F. Tetrahedron: Asymmetry 2002, 13, 243; (d) Zhu, Y.; Chen, L.; Kong, F. Carbohydr. Res. 2002, 337, 207.

15. (a) Zhang, J.; Kong, F. J. Carbohydr. Chem. 2002, 21, 79;

(b) Zhang, J.; Kong, F. Carbohydr. Res. 2002, 337, 391;

(c) Zhang, J.; Zhu, Y.; Kong, F. Carbohydr. Res. 2001, 336, 329 .

16. Sheng, S.; Cherniak, R. Carbohydr. Res. 1997, 301, 33.

17. Copeland, C.; Stick, R. V. Aust. J. Chem. 1978, 31, 1371.

18. Schmidt, R. R.; Kinzy, W. Adv. Carbohydr. Chem. Biochem. 1994, 50, 21-125.

19. Byramova, N. E.; Ovchinnikov, M. V.; Backinowsky, L. V.; Kochetkov, N. K. Carbohydr. Res. 1983, 124, c8.

20. All new compounds gave satisfactory elemental analysis results. Selected physical data for some key compounds are as follows, for 8: $[\alpha]_{\mathrm{D}}+91.7\left(c 1.0, \mathrm{CHCl}_{3}\right) ;{ }^{1} \mathrm{H} \mathrm{NMR}$ $\left(\mathrm{CDCl}_{3}, 400 \mathrm{MHz}\right): \delta$ 7.95-7.34 (m, $\left.15 \mathrm{H}, 3 \mathrm{PhH}\right), 5.94$ $\left(\mathrm{dd}, 1 \mathrm{H}, J_{3,4}=J_{4,5}=10.0 \mathrm{~Hz}, \mathrm{H}-4\right), 5.75(\mathrm{dd}, 1 \mathrm{H}$, $\left.J_{2,3}=3.3 \mathrm{~Hz}, J_{3,4}=10.0 \mathrm{~Hz}, \mathrm{H}-3\right), 5.53\left(\mathrm{dd}, 1 \mathrm{H}, J_{1,2}=1.8\right.$ $\left.\mathrm{Hz}, J_{2,3}=3.3 \mathrm{~Hz}, \mathrm{H}-2\right), 5.43\left(\mathrm{~d}, 1 \mathrm{H}, J_{1,2}=1.8 \mathrm{~Hz}, \mathrm{H}-1\right)$, $5.31\left(\mathrm{~d}, 1 \mathrm{H}, J_{1,2}=1.0 \mathrm{~Hz}, \mathrm{H}-1\right), 4.71\left(\mathrm{~d}, 1 \mathrm{H}, J_{2,1}=1.0\right.$ $\mathrm{Hz}, \mathrm{H}-1), 4.66-4.58$ (m, $3 \mathrm{H}), 4.26-4.18$ (m, $4 \mathrm{H}), 4.04$ $3.99(\mathrm{~m}, 2 \mathrm{H}), 3.90-3.82(\mathrm{~m}, 4 \mathrm{H}), 3.68-3.63(\mathrm{~m}, 2 \mathrm{H})$, $3.36\left(\mathrm{~s}, 3 \mathrm{H}, \mathrm{OCH}_{3}\right), 2.17$ (s, $\left.3 \mathrm{H}, \mathrm{COCH}_{3}\right), 1.56,1.45$ (2s, $6 \mathrm{H}$, isopropylidene), $1.37\left(\mathrm{~s}, 6 \mathrm{H}\right.$, isopropylidene). ${ }^{13} \mathrm{C}$ NMR $\left(100 \mathrm{MHz}, \mathrm{CDCl}_{3}\right): 169.5\left(\mathrm{COCH}_{3}\right), 166.3,165.6$, 163.6 (5 C, $5 \mathrm{COPh}), 101.2,100.8$ (2 C, $\left.2 \mathrm{Me}_{2} C\right)$, 99.9, 99.4, 98.3 (3 C, $3 \mathrm{C}-1), 54.8\left(\mathrm{OCH}_{3}\right), 29.1,28.9$ (2 C, $\left.\mathrm{CH}_{3} \mathrm{CCH}_{3}\right), 20.6\left(\mathrm{COCH}_{3}\right), 19.2,19.0\left(2 \mathrm{C}, \mathrm{CH}_{3} \mathrm{CCH}_{3}\right)$. Anal. calcd for $\mathrm{C}_{48} \mathrm{H}_{56} \mathrm{O}_{20}: \mathrm{C}, 60.50 ; \mathrm{H}, 5.92$. Found: $\mathrm{C}$, 60.45; H, 5.66. For 9: $[\alpha]_{\mathrm{D}}+82.6\left(c 1.0, \mathrm{CHCl}_{3}\right) ;{ }^{1} \mathrm{H}$ NMR $\left(\mathrm{CDCl}_{3}, 400 \mathrm{MHz}\right): \delta 8.10-7.23(\mathrm{~m}, 15 \mathrm{H}, 3 \mathrm{PhH})$, $6.04\left(\mathrm{dd}, 1 \mathrm{H}, J_{3,4}=J_{4,5}=10.0 \mathrm{~Hz}, \mathrm{H}-4\right), 5.64(\mathrm{dd}, 1 \mathrm{H}$, $\left.J_{2,3}=3.2 \mathrm{~Hz}, J_{3,4}=10.0 \mathrm{~Hz}, \mathrm{H}-3\right), 5.45\left(\mathrm{dd}, 1 \mathrm{H}, J_{1,2}=2.0\right.$ $\left.\mathrm{Hz}, J_{2,3}=2.9 \mathrm{~Hz}, \mathrm{H}-2\right), 5.40\left(\mathrm{dd}, 1 \mathrm{H}, J_{1,2}=1.3 \mathrm{~Hz}\right.$, $\left.J_{2,3}=3.4 \mathrm{~Hz}, \mathrm{H}-2\right), 5.32\left(\mathrm{~d}, 1 \mathrm{H}, J_{1,2}=1.7 \mathrm{~Hz}, \mathrm{H}-1\right), 5.22$ $\left(\mathrm{dd}, 1 \mathrm{H}, J_{1,2}=1.3 \mathrm{~Hz}, J_{2,3}=2.9 \mathrm{~Hz}, \mathrm{H}-2\right), 5.10(\mathrm{~d}, 1 \mathrm{H}$, $\left.J_{1,2}=1.2 \mathrm{~Hz}, \mathrm{H}-1\right), 4.67\left(\mathrm{dd}, 1 \mathrm{H}, J_{2,3}=2.9 \mathrm{~Hz}, J_{3,4}=12.1\right.$ $\mathrm{Hz}, \mathrm{H}-3), 4.63$ (d, $\left.1 \mathrm{H}, J_{1,2}=1.2 \mathrm{~Hz}, \mathrm{H}-1\right), 4.46-4.38$ (m, $2 \mathrm{H}), 4.09-4.05(\mathrm{~m}, 4 \mathrm{H}), 3.87-3.81(\mathrm{~m}, 4 \mathrm{H}), 3.70-3.60$ $(\mathrm{m}, 2 \mathrm{H}), 3.36\left(\mathrm{~s}, 3 \mathrm{H}, \mathrm{OCH}_{3}\right), 2.31,2.18,2.07(3 \mathrm{~s}, 9 \mathrm{H}$, $\left.3 \mathrm{COCH}_{3}\right), 1.56,1.52(2 \mathrm{~s}, 6 \mathrm{H}$, isopropylidene), 1.37 (s, $6 \mathrm{H}$, isopropylidene). Anal. calcd for $\mathrm{C}_{52} \mathrm{H}_{60} \mathrm{O}_{22}: \mathrm{C}, 60.22$; $\mathrm{H}$, 5.83. Found: $\mathrm{C}, 60.47$; H, 5.61. For 12: $[\alpha]_{\mathrm{D}}-58.6(c$
1.0, $\left.\mathrm{CHCl}_{3}\right) ;{ }^{1} \mathrm{H} \mathrm{NMR}\left(\mathrm{CDCl}_{3}, 400 \mathrm{MHz}\right): \delta$ 8.11-7.34 $(\mathrm{m}, 65 \mathrm{H}, 13 \mathrm{PhH}), 5.94\left(\mathrm{dd}, 1 \mathrm{H}, J_{3,4}=J_{4,5}=10.0 \mathrm{~Hz}\right.$, $\mathrm{H}-4, \operatorname{Man} p), 5.77\left(\mathrm{dd}, 1 \mathrm{H}, J_{2,3}=3.2 \mathrm{~Hz}, J_{3,4}=10.0 \mathrm{~Hz}\right.$, H-3, Manp), $5.71\left(\mathrm{dd}, J_{1,2}=J_{2,3}=6.3 \mathrm{~Hz}, \mathrm{H}-2, \mathrm{Xyl} p\right)$, $5.59\left(\mathrm{dd}, 1 \mathrm{H}, J_{3,4}=J_{4,5}=10.0 \mathrm{~Hz}, \mathrm{H}-4, \operatorname{Man} p\right), 5.54(\mathrm{dd}$, $\left.1 \mathrm{H}, J_{3,4}=J_{4,5}=9.9 \mathrm{~Hz}, \mathrm{H}-4, \operatorname{Man} p\right), 5.47-5.40(\mathrm{~m}, 3 \mathrm{H})$, 5.31-5.27 (m, $2 \mathrm{H}), 5.18\left(\mathrm{~d}, 1 \mathrm{H}, J_{1,2}=0.7 \mathrm{~Hz}, \mathrm{H}-1\right.$, $\operatorname{Man} p), 5.16\left(\mathrm{dd}, 1 \mathrm{H}, J_{1,2}=1.0 \mathrm{~Hz}, J_{2,3}=3.0 \mathrm{~Hz}, \mathrm{H}-2\right.$, $\operatorname{Man} p), 4.99$ (d, $\left.1 \mathrm{H}, J_{1,2}=0.8 \mathrm{~Hz}, \mathrm{H}-1, \operatorname{Man} p\right), 4.80$ (d, $\left.1 \mathrm{H}, J_{1,2}=4.8 \mathrm{~Hz}, \mathrm{H}-1, \mathrm{Xyl} p\right), 4.53\left(\mathrm{~d}, 1 \mathrm{H}, J_{1,2}=1.0 \mathrm{~Hz}\right.$, H-1, Manp), 4.45 (d, $\left.1 \mathrm{H}, J_{1,2}=6.1 \mathrm{~Hz}, \mathrm{H}-1, \mathrm{Xyl} p\right), 3.19$ $\left(\mathrm{s}, 3 \mathrm{H}, \mathrm{OCH}_{3}\right), 1.90\left(\mathrm{~s}, 3 \mathrm{H}, \mathrm{COCH}_{3}\right),{ }^{13} \mathrm{C} \mathrm{NMR}(100$ $\left.\mathrm{MHz}, \mathrm{CDCI}_{3}\right)$ : $168.9\left(\mathrm{COCH}_{3}\right), 166.0,165.9,165.8$, $165.4,165.3,165.3,165.3,165.2,165.1,165.0,164.9$, 164.7, 164.6 (13 C, $13 \mathrm{COPh}), 99.7,99.7,99.7,99.0,98.5$ (5 C, $5 \mathrm{C}-1), 54.8\left(\mathrm{OCH}_{3}\right), 20.3\left(\mathrm{COCH}_{3}\right)$; Anal. calcd for $\mathrm{C}_{122} \mathrm{H}_{104} \mathrm{O}_{38}$ : C, 67.27; H, 4.81. Found: C, 67.45; H, 5.96. For 15: $[\alpha]_{\mathrm{D}}-22.5\left(\right.$ c $\left.0.5, \mathrm{CHCl}_{3}\right) ;{ }^{1} \mathrm{H} \mathrm{NMR}\left(\mathrm{CDCl}_{3}, 400\right.$ $\mathrm{MHz}): \delta 8.13-7.30(\mathrm{~m}, 65 \mathrm{H}, 13 \mathrm{Ph} H), 6.00(\mathrm{dd}, 1 \mathrm{H}$, $\left.J_{3,4}=J_{4,5}=10.0 \mathrm{~Hz}, \mathrm{H}-4, \operatorname{Man} p\right), 5.71\left(\mathrm{dd}, 1 \mathrm{H}, J_{2,3}=3.2\right.$ $\left.\mathrm{Hz}, J_{3,4}=10.0 \mathrm{~Hz}, \mathrm{H}-3, \operatorname{Man} p\right), 5.15\left(\mathrm{~d}, 1 \mathrm{H}, J_{1,2}=1.0\right.$ $\mathrm{Hz}, \mathrm{H}-1, \operatorname{Man} p), 4.96$ (d, $1 \mathrm{H}, J_{1,2}=0.9 \mathrm{~Hz}, \mathrm{H}-1, \operatorname{Man} p$ ), $4.75\left(\mathrm{~d}, 1 \mathrm{H}, J_{1,2}=4.9 \mathrm{~Hz}, \mathrm{H}-1, \mathrm{Xyl} p\right), 4.60(\mathrm{~d}, 1 \mathrm{H}$, $\left.J_{1,2}=4.8 \mathrm{~Hz}, \mathrm{H}-1, \mathrm{Xyl} p\right), 4.56\left(\mathrm{~d}, 1 \mathrm{H}, J_{1,2}=1.0 \mathrm{~Hz}, \mathrm{H}-1\right.$, $\operatorname{Man} p), 4.04\left(\mathrm{~d}, 1 \mathrm{H}, J_{1,2}=6.6 \mathrm{~Hz}, \mathrm{H}-1\right.$, GluAp), 3.69 (s, $\left.3 \mathrm{H}, \mathrm{COOCH}_{3}\right), 3.23\left(\mathrm{~s}, 3 \mathrm{H}, \mathrm{OCH}_{3}\right), 1.96,1.92,1.31(3 \mathrm{~s}$, $\left.9 \mathrm{H}, 3 \mathrm{COCH}_{3}\right) \cdot{ }^{13} \mathrm{C}$ NMR $\left(100 \mathrm{MHz}, \mathrm{CDCl}_{3}\right): 167.0$, $168.5,168.5,168.3$ ( $\left.3 \mathrm{C}, 3 \mathrm{COCH}_{3}, C \mathrm{OOM}\right), 165.9$, $165.9,165.9,165.4,165.4,165.4,165.3,165.2,165.1$, 165.0, 164.9, 164.6, 164.6 (13 C, $13 \mathrm{COPh}), 100.9$ (C-1, $\left.J_{\mathrm{C} 1, \mathrm{H} 1}=175 \mathrm{~Hz}, \operatorname{Man} p\right), 100.3\left(\mathrm{C}-1, J_{\mathrm{C} 1, \mathrm{H} 1}=163 \mathrm{~Hz}\right.$, GluAp $), 99.9\left(\mathrm{C}-1, J_{\mathrm{C} 1, \mathrm{H} 1}=164 \mathrm{~Hz}, \mathrm{Xyl} p\right), 99.5(\mathrm{C}-1$, $\left.J_{\mathrm{C} 1, \mathrm{H} 1}=163 \mathrm{~Hz}, \mathrm{Xyl} p\right), 98.5\left(\mathrm{C}-1, J_{\mathrm{C} 1, \mathrm{H} 1}=172 \mathrm{~Hz}\right.$, $\operatorname{Man} p), 95.2\left(\mathrm{C}-1, J_{\mathrm{C} 1, \mathrm{H} 1}=176 \mathrm{~Hz}, \operatorname{Man} p\right), 54.8\left(\mathrm{OCH}_{3}\right)$, $52.3\left(\mathrm{COOCH}_{3}\right), 20.5,20.3,20.2\left(\mathrm{COCH}_{3}\right)$; Anal. Calcd for $\mathrm{C}_{133} \mathrm{H}_{118} \mathrm{O}_{46}: \mathrm{C}, 65.13 ; \mathrm{H}, 4.85$. Found: $\mathrm{C}, 65.45 ; \mathrm{H}$, 4.66. For 16: $[\alpha]_{\mathrm{D}}+99.6\left(c 0.5, \mathrm{H}_{2} \mathrm{O}\right) ;{ }^{1} \mathrm{H} \mathrm{NMR}\left(\mathrm{D}_{2} \mathrm{O}\right.$, $400 \mathrm{MHz}$ ): $\delta 5.11$ (s, $1 \mathrm{H}, \mathrm{H}-1, \operatorname{Man} p$ ), 4.75 (s, $1 \mathrm{H}, \mathrm{H}-1$, $\operatorname{Man} p), 4.31(\mathrm{~s}, 1 \mathrm{H}, \mathrm{H}-1, \operatorname{Man} p), 4.29\left(\mathrm{~d}, 1 \mathrm{H}, J_{1,2}=8.0\right.$ $\mathrm{Hz}, \mathrm{H}-1$, GluAp), 4.12 (d, $1 \mathrm{H}, J_{1,2}=8.9 \mathrm{~Hz}, \mathrm{H}-1, \mathrm{Xyl} p$ ), $4.10\left(\mathrm{~d}, 1 \mathrm{H}, J_{1,2}=9.2 \mathrm{~Hz}, \mathrm{H}-1, \mathrm{Xyl} p\right), 3.33(\mathrm{~s}, 3 \mathrm{H}$, $\left.\mathrm{OCH}_{3}\right) ;{ }^{13} \mathrm{C}$ NMR $\left(100 \mathrm{MHz}, \mathrm{D}_{2} \mathrm{O}\right): 174.1\left(-\mathrm{COONH}_{4}\right)$, 103.4, 103.3, 103.2, 102.5, 100.3, 100.2 (6 C-1), 79.0, 78.5, $78.4,78.3,78.3,76.4,76.2,75.9,75.8,73.6,73.4,73.2$, $72.9,72.9,70.7,70.3,69.5,69.5,68.4,67.0,66.7,66.5$, 65.4, 65.3, 61.5, 60.6, 60.6, $56.6\left(\mathrm{O}-\mathrm{CH}_{3}\right)$. MALDI-TOF MS calcd for the ammonium salt of $16, \mathrm{C}_{35} \mathrm{H}_{61} \mathrm{O}_{30} \mathrm{~N}$ : 975.8 [M]. found: $975.8(\mathrm{M})$; $980.9\left(\mathrm{M}-\mathrm{NH}_{4}^{+}+\mathrm{Na}^{+}\right)$. 